\title{
Local vs. wide-area undervoltage load shedding in the presence of induction motor loads
}

\author{
Bogdan Otomega Thierry Van Cutsem, Fellow, IEEE
}

\begin{abstract}
In the presence of induction motor loads, long-term voltage instability may end up in a sharp voltage decrease, which makes the tuning of undervoltage load shedding more delicate. This paper first investigates the ability of a purely distributed load shedding scheme, previously proposed by the authors, to cope with these situations. Owing to difficulties to reconcile dependability and security, an alternative wide-area protection is considered. The latter consists of generators sending overexcitation signals to the load shedding controllers in order to allow the latter to act faster. Detailed time simulations of a test system are reported.
\end{abstract}

Index Terms-Long-term voltage instability, induction motors, undervoltage load shedding, distributed control, wide-area protection

\section{INTRODUCTION}

$\mathbf{I}^{\mathrm{T}}$ $\mathrm{T}$ is known for quite some time that load shedding is a cost-effective countermeasure against voltage instability triggered by large disturbances [1], [2], [3]. It is needed when preventive actions are not economically justified due the low occurrence probability of severe disturbances or when the initial voltage drop is too pronounced to be corrected by other means. Although some event-based schemes have been successfully devised [4], a majority of schemes are of the response-based type [5], [6], [7], allowing to adjust the corrective action to the disturbance severity and to operate in closed loop for higher robustness.

This paper is the continuation of Ref. [8], where a distributed undervoltage load shedding scheme was proposed to protect against long-term voltage instability. This scheme relies on a set of distributed controllers covering the region prone to voltage instability. Each controller monitors the voltage at a transmission bus and acts on a set of loads electrically close to that bus. This allows the protection to adjust to the disturbance location. Furthermore, this scheme operates in closed-loop, adjusting the amount of load shed to the severity of the disturbance. Its robustness against system behaviour uncertainties and operation failures is guaranteed by the redundancy among the controllers. The latter are coordinated through the network voltages, without resorting to a dedicated communication link. More recently, Ref. [9] reported on tests performed on a large-scale model of real system, including coordination with tap changer blocking.

B. Otomega (bogdan.otomega@yahoo.com) is senior lecturer in the Power Systems Dept. of the "Politehnica" University of Bucharest, Romania.

T. Van Cutsem (t.vancutsem@ulg.ac.be) is a research director of the Fund for Scientific Research (FNRS) and adjunct professor in the Dept. of Electrical Engineering and Computer Science (Montefiore Institute) of the University of Liège, Belgium.

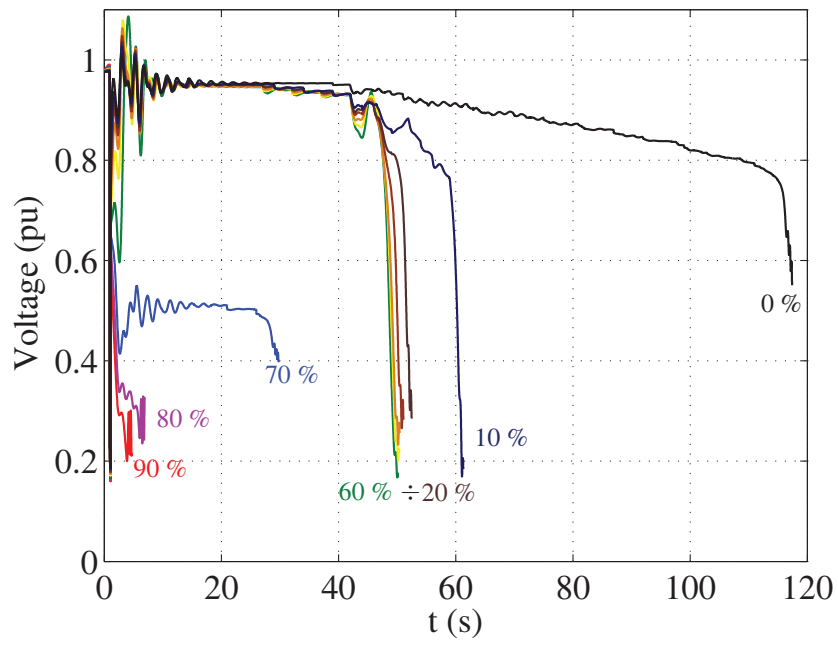

Fig. 1. Post-disturbance voltage evolution for various proportions of induction motor load

This paper deals with the impact of induction motor loads on the load shedding design. The objective of the paper is twofold: first, investigate how far the above distributed scheme can cope with the fast responding induction motor loads; second, consider an alternative scheme that bears the spirit of wide-area protection by using additional information from neighbouring OverExcitation Limiter (OEL) activation, in the same spirit as [5].

As is well-known, induction motors may precipitate voltage instability owing to their trend to restore the air-gap power to a (nearly) constant value, irrespective of the network voltage [10], [11].

Induction motors play an important rôle in short-term voltage instability, where the main concern is their ability to reaccelerate after a fault. When this is not possible, motors stall, and large currents are drawn from the system, causing voltages to sag and other motors to stall as well. Fast undervoltage load shedding can be an effective countermeasure. Induction motors also impact long-term instability by making transmission voltages drop sharply once some key generators stop supporting transmission voltages, under the effect of field current limitation.

Both aspects are illustrated in Fig. 1, relative to the test system considered later on in this paper. The curves show the evolution of a transmission voltage under the effect of a fault cleared by permanently opening a line. In the absence of induction motors, the voltage drops progressively under the effect of OELs and Load Tap Changer (LTCs), until the system 


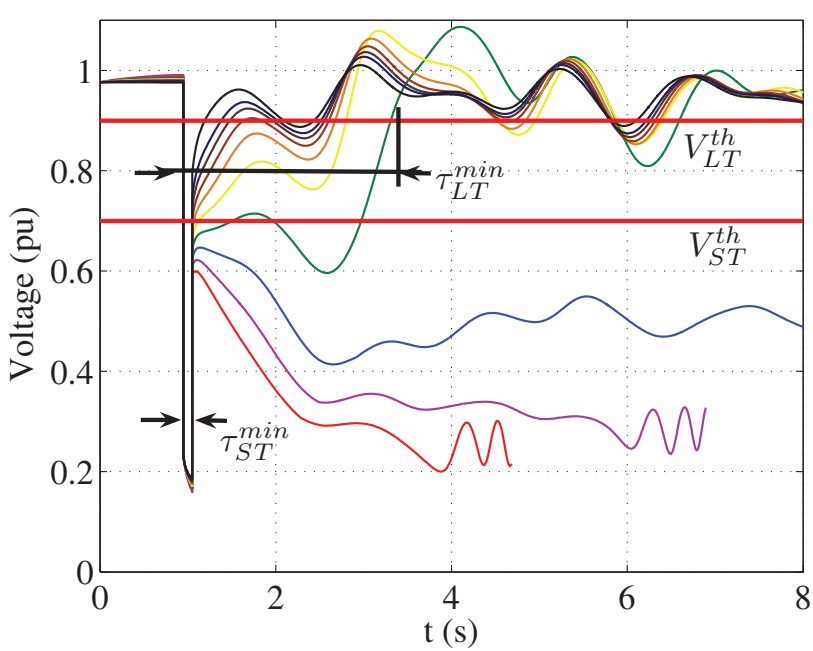

Fig. 2. Typical voltage thresholds and minimum shedding delays for shortand long-term unstable cases

collapses at $t \simeq 117 \mathrm{~s}$ owing to field current limited generators loosing synchronism. When a little proportion (as small as $10 \%$ ) of motor loads is assumed, the voltage collapses earlier but, more importantly, the fall is significantly sharper. This drop is caused by the stalling of induction motors. In this case, it becomes more difficult for a load shedding scheme to rely on the voltage magnitudes only. When the proportion of motors increases to $70 \%$, the instability becomes of the short-term type, with the motors stalling under the effect of the initial fault itself.

The paper is organized as follows. Section II recalls the principle of the distributed scheme, describes its potential limitations in the presence of induction motors and introduces the wide-area protection scheme. Simulation results relative to both schemes are presented in Section III and IV, respectively. Section V offers some conclusion.

\section{UNDERVOLTAGE LOAD SHEDDING SCHEMES}

\section{A. Short vs. long-term settings}

Two essential settings of an undervoltage load shedding scheme are the voltage threshold $V^{t h}$ below which the controller is started, and the delay $\tau$ before loads are effectively disconnected. Different settings must be used to deal with short- and long-term voltage instabilities, respectively. This is discussed hereafter, and illustrated by Fig. 2, showing the voltage evolutions of Fig. 1 over the first 8 seconds.

In response to motor stalling or slow re-acceleration after a fault, load shedding should be actuated at a low voltage, typically $V_{S T}^{t h} \simeq 0.7 \mathrm{pu}$, as shown in the figure. The corresponding delay $\tau_{S T}$ should be as short as possible, but large enough to avoid shedding after a normal fault clearing followed by a stable recovery of voltages.

The mechanism leading to long-term voltage instability is different [11] and usually requires a higher value of the voltage threshold $V_{L T}^{t h}$, typically in the range [ $\left.\begin{array}{ll}0.8 & 0.9\end{array}\right]$ pu (or even higher for capacitive transmission systems [6]). Lower thresholds would delay the shedding and, hence, would require shedding more to stabilize the system, or could even fail saving the system. The main consequence of this higher threshold value is easily seen from Fig. 2: due to the post-disturbances swings, a significantly larger delay $\tau_{L T}^{\min }$ is required to avoid shedding unduly in stable cases. This delay, however, has the same drawback as setting $V_{L T}^{t h}$ to a lower value.

This paper further discusses the choice of $\left(V_{L T}^{t h}, \tau_{L T}\right)$ to counteract long-term voltage instability, and proposes an alternative design in case no satisfactory setting can be found. For simplicity, the ${ }_{L T}$ lowerscript is dropped.

If both short- and long-term problems are anticipated, the two rules may coexist, the short-term one possibly serving as backup for the long-term one.

\section{B. Distributed protection scheme}

We first consider the distributed load shedding scheme detailed in [8], [9]. It relies on a set of controllers covering the region prone to voltage instability. Each controller monitors a transmission voltage $V$ and compares the latter to the threshold $V^{t h}$. If a disturbance causes $V$ to become smaller than $V^{t h}$, the controller is started and after a time delay $\tau$ it sheds a load power $\Delta P_{s h}$ by opening distribution circuit breakers. The controller returns to idle state as soon as the voltage recovers above the threshold. To this purpose, the controller can act several times, within the limits of interruptible load power, of course.

The values of both $\tau$ and $\Delta P_{s h}$ depend on the dynamic evolution of $V$, via two parameters, $C$ and $K$, as follows:

$$
\begin{gathered}
\int_{t_{0}}^{t_{0}+\tau}\left(V^{t h}-V(t)\right) d t=C \quad \text { with } \quad \tau \geq \tau_{\text {min }} \\
\Delta P^{s h}=K \cdot \frac{1}{\tau} \int_{t_{0}}^{t_{0}+\tau}\left(V^{t h}-V(t)\right) d t
\end{gathered}
$$

where $t_{0}$ is the time at which the controller is started, and $\tau_{\text {min }}$ corresponds to the minimum delay discussed in the previous section. A larger value of $C$ leads a controller to waiting longer before it can shed load, while a larger value of $K$ leads to shedding more, for the same average voltage drop.

When designing the controllers from a set of scenarios, the values of $C, K$ and $V^{t h}$ are chosen so that [8], [9]:

- no load is shed in stable cases;

- all unstable cases are saved;

- as few load as possible is shed when needed;

- the nuisance of low voltages on customers is minimized.

\section{Wide-area protection scheme}

As already discussed, the fast response of induction motors requires to decrease the value of $\tau$, while the security of the protection scheme requires to keep $\tau$ above a certain value. These conflicting requirements can be met by resorting to additional information that allows the distributed shedding controllers to act faster when appropriate. To this purpose, information is going to be sent from generators to load shedding controllers and the protection scheme will change from purely distributed to wide-area, although simplicity will be preserved. 


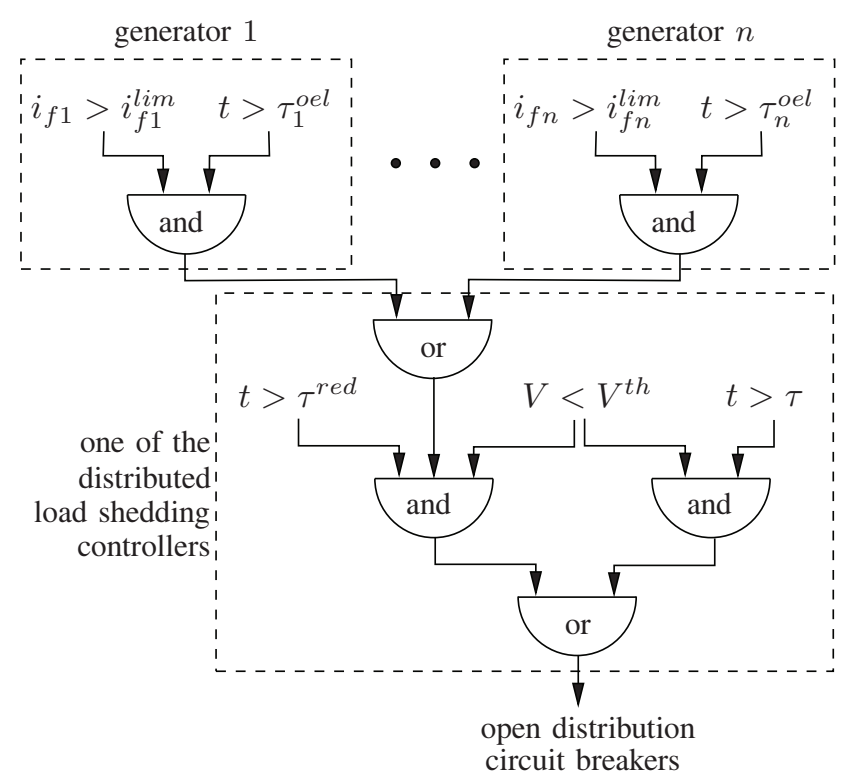

Fig. 3. Logic of the wide-area protection scheme

In long-term unstable situations, motor stalling typically results from the fall of transmission voltages induced by the activation of generator OELs. Hence, the idea is to inform the load shedding controllers that the field currents of neighbouring generators exceed the limit supervised by their OELs, and hence are going to be reduced to this value (after the OEL activation time is elapsed). Upon receipt of this information, the load shedding controllers reduce their activation delay to a much smaller value so that, if the monitored voltage indeed falls below the threshold, load shedding takes place much more rapidly.

Since a generator field current may temporarily exceed its OEL limit, under the effect of a nearby fault, the current has to remain above the limit for some time $\tau^{\text {oel }}$ before sending the signal, so that the latter truly reveals a situation where the generator is overexcited owing to its voltage control.

A graphical representation of the logic is given in Fig. 3. $\tau$ is the load shedding delay considered in the previous section while $\tau^{r e d}$ is the shorter delay used upon receipt of the OEL signal from at least one of the generators in the vicinity of the controller. The field current $i_{f i}$ of the $i$-th generator has to remain above the OEL limit $i_{f i}^{l i m}$ for the duration $\tau_{i}^{o e l}$ before that generator sends its overexcitation signal. Of course, it is still required to have $V<V^{\text {th }}$ in order load shedding to be triggered. Following a normally cleared fault, this condition is fulfilled but, as the generator signal has not been received, the longer delay $\tau$ will be in effect. Hence, provided that $\tau$ has been set to a value larger than $\tau_{L T}^{\min }$ in Fig. 2, no shedding will take place. Finally, as a back-up in case of failure to receive the generator signals, load shedding takes place with the longer delay $\tau$.

\section{SimUlation RESULTS: DistRIBUTED SCHEME}

\section{A. Test system}

The proposed scheme has been tested on a variant of the Nordic32 test system, already used in [12], [13]. The system

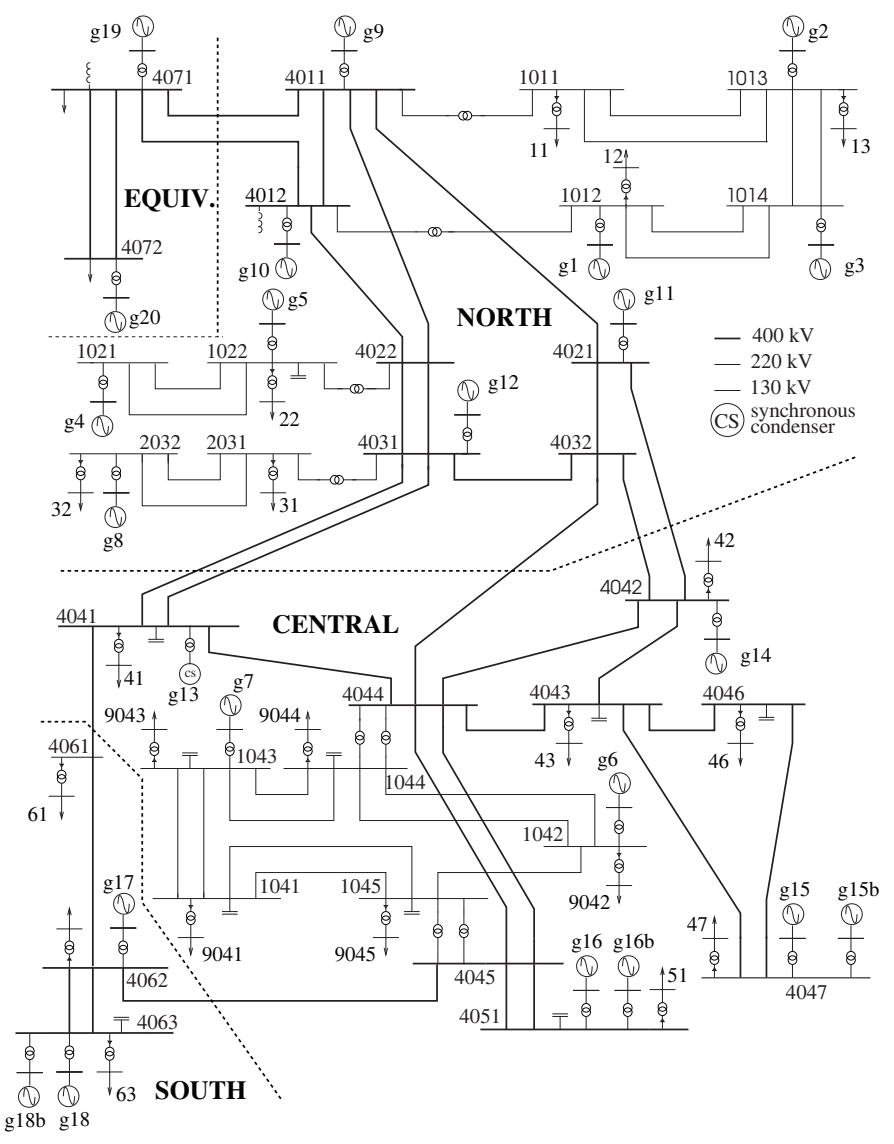

Fig. 4. Nordic32 test system

one-line diagram is shown in Fig. 4. The model includes 55 buses, 23 generators, and 22 loads. Detailed time-domain simulations have been used as in [12], [13]. The long-term dynamics are driven by LTCs and OELs acting with various delays. When generator excitations are relieved by load shedding, the OELs reset and the Automatic Voltage Regulators regain control with negligible delay.

Since voltage problems are experienced in the "Central" region, the latter has been provided with five load shedding controllers, as detailed in Table I. Each controller monitors the voltage of one transmission bus and controls the load on the distribution side of the transformer connected to that bus.

TABLE I

CONSIDERED CONTROLLERS AND LOAD COMPOSITION

\begin{tabular}{|c|c|c|c|c|}
\hline $\begin{array}{c}\text { Controller } \\
\text { name }\end{array}$ & $\begin{array}{c}\text { Monitored } \\
\text { bus }\end{array}$ & $\begin{array}{c}\text { Controlled } \\
\text { load bus }\end{array}$ & $\begin{array}{c}\text { Exponential } \\
\text { load (MW) }\end{array}$ & $\begin{array}{c}\text { Induction motor } \\
\text { load (MW) }\end{array}$ \\
\hline$C_{1041}$ & 1041 & 9041 & 300 & 300 \\
\hline$C_{1042}$ & 1042 & 9042 & 150 & 150 \\
\hline$C_{1043}$ & 1043 & 9043 & 115 & 115 \\
\hline$C_{1044}$ & 1044 & 9044 & 400 & 400 \\
\hline$C_{1045}$ & 1045 & 9045 & 350 & 350 \\
\hline
\end{tabular}

All loads in the "Central" area are represented with an exponential model for half of the load power and an equivalent induction motor for the other half (see Table I). The former part behaves as constant current for active power and constant impedance for reactive power.

It has been assumed that only the exponential part of the 


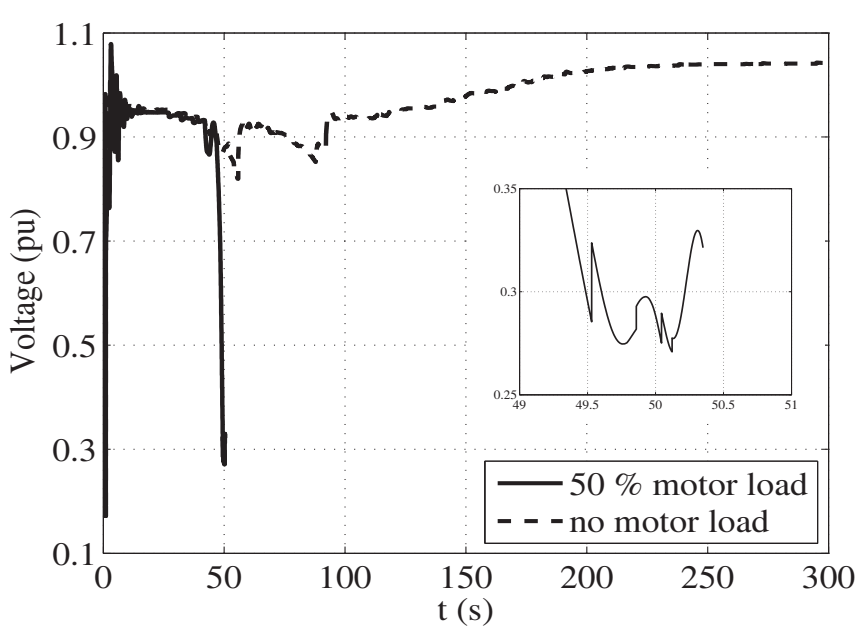

Fig. 5. Evolution of voltage at bus 1041 with 0 and $50 \%$ of motor load

loads can be shed. This makes the tests more severe since the whole induction motor load remains connected to the system.

\section{B. Impact of motor loads on load shedding performance}

The results shown hereafter deal with the system response to a 3-phase fault occurring on line 4032-4044, cleared in 0.1 second by opening the line, which remains disconnected. Due to a heavy power transfer from North to South, the predisturbance operating point is not (N-1)-secure and the line outage causes long-term voltage instability as shown in Fig. 1.

First, an exponential load model has been considered (0\% motor load) and the load shedding controllers have been tuned according to the methodology described in [8]. For the considered set of contingencies, the best settings are: $V^{\text {th }}=0.90 \mathrm{pu}, C=0.3 \mathrm{pu} \cdot \mathrm{s}, K=4000 \mathrm{MW} / \mathrm{pu}$. $\tau_{\text {min }}$ has been set to $3 \mathrm{~s}$ to avoid reacting to a normally cleared fault.

In the absence of load shedding, the system is unstable and the voltage at bus 1041 evolves as shown by the rightmost curve in Fig. 1, resulting in a collapse at $t \simeq 117 \mathrm{~s}$. The corresponding evolution in the presence of the distributed load shedding controllers is shown with dotted line in Fig. 5. The system is stabilized in a quite satisfactory way.

Next, $50 \%$ of the loads are assumed to be of the motor type. In this case, without load shedding, the system collapses at $t \simeq 50 \mathrm{~s}$, as was shown in Fig. 1. The field currents of three generators located close to the Central region are shown in Fig. 6. The OELs of g14, g12 and g15 act successively. When the last limitation takes effect, motors stall and voltage collapses. The corresponding voltage evolution in the presence of the distributed load shedding controllers, with the same settings as in the no-motor case, is shown with solid line in Fig. 5. The controllers fail saving the system. In fact, the above values of $C$ and $\tau_{\min }$ make them react too slowly (the zoom in Fig. 5 unveils their late, useless responses).

\section{Setting the controller parameters to cope with motor loads}

To make the controllers react faster, it was chosen to leave Eq. (1) and make the protection operate with a fixed, as small as possible delay $\tau$. As already discussed, this value has to be

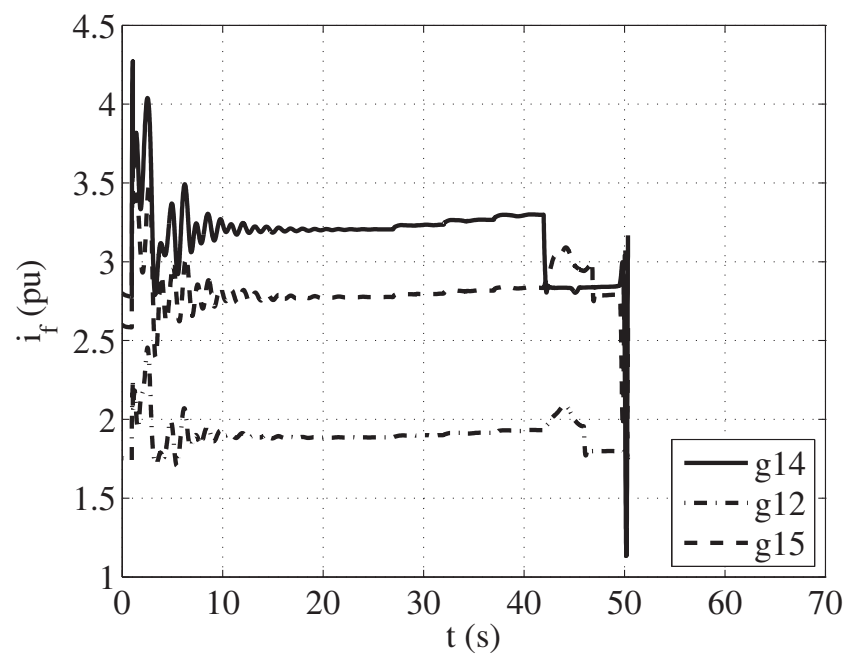

Fig. 6. Evolution of field currents of generators located close to Central area

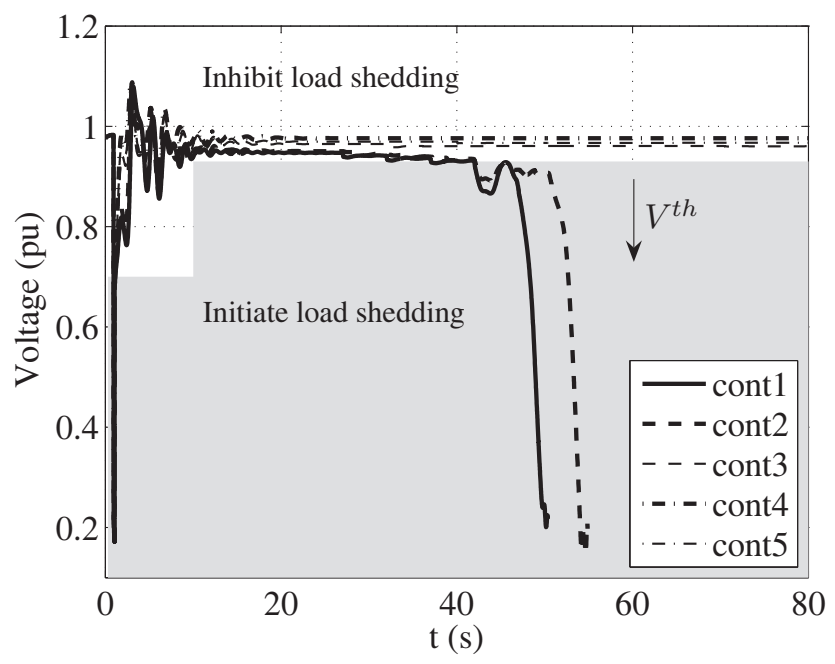

Fig. 7. Voltage evolution for five contingencies

large to prevent the controller from reacting on a nearby fault. The proper value depends on the chosen voltage threshold $V^{t h}$. On one hand, $V^{t h}$ should be set high enough to avoid excessive shedding delays (which would require to shed more and/or cause low load voltages). On the other hand, $V^{\text {th }}$ should be set below the lowest voltage value experienced during any of the acceptable post-disturbance evolutions. This is illustrated in Fig. 7 for various contingencies and in Fig. 8 for different predisturbance loading conditions. The protection scheme should operate in the greyed areas of these figures.

The analysis of various post-disturbance evolutions, such as the ones presented in Figs. 7 and 8, leads to taking $V^{t h}<0.91 \mathrm{pu}$, as a compromise between dependability and selectivity of the protection. Indeed, this leaves some margin with respect to the lowest voltage observed during $\mathrm{N}$ 1 contingencies without affecting the protection performance. For $V^{t h}=0.90 \mathrm{pu}$, for instance, $\tau$ should be set to at least $2 \mathrm{~s}$, in order not to react to the initial fault, as shown in Fig. 9. Hence, tests were performed for values of $\tau$ in the interval $\left[\begin{array}{ll}2 & 3\end{array}\right] \mathrm{s}$. 


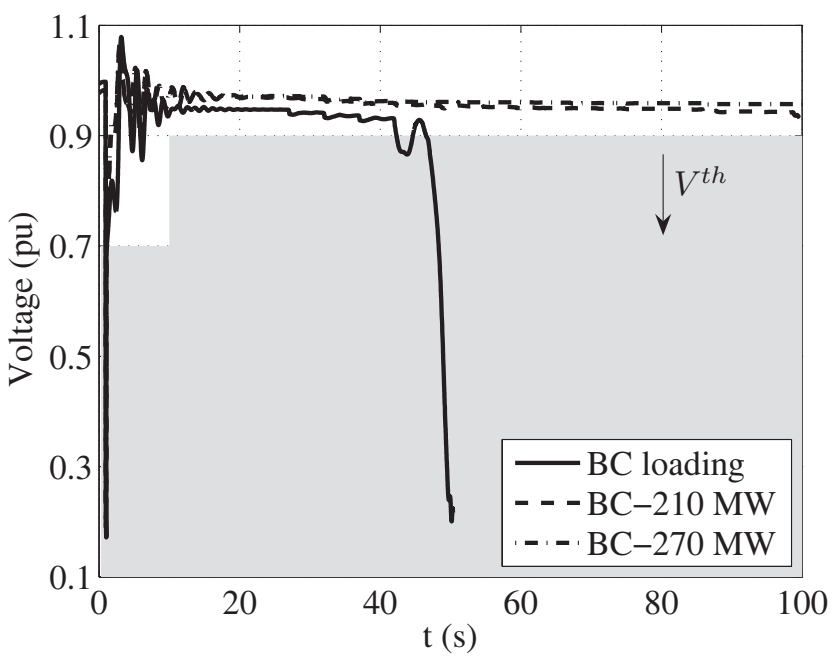

Fig. 8. Voltage evolution for various pre-contingency load levels. The disturbance "cont1" in Fig. 7 is considered; BC denotes the base case situation

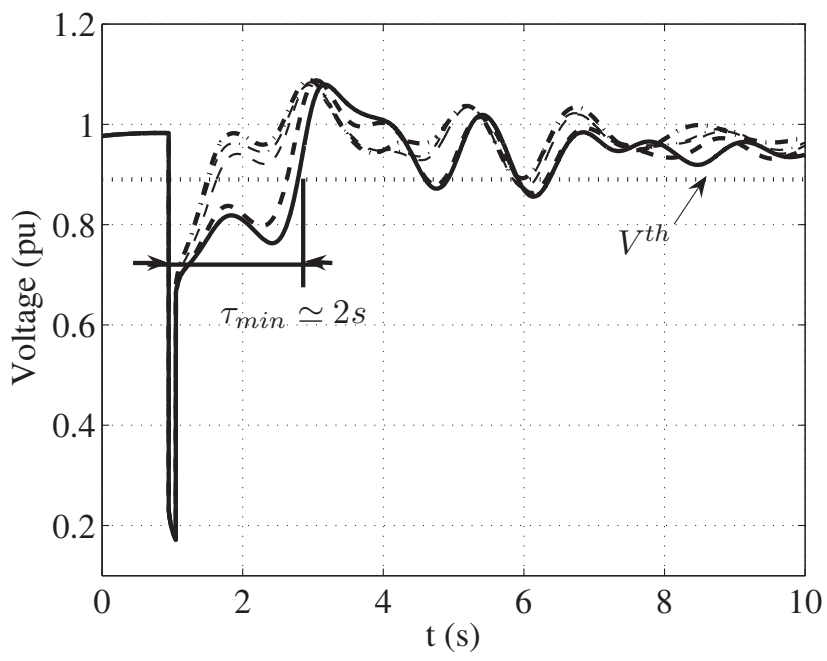

Fig. 9. Voltage evolution for different contingencies

As for parameter $K$ in (2), its value should be selected so that the protection sheds as few load as possible over all the scenarios [8], [9].

Figure 10 presents the performance of the load shedding scheme for various $V^{t h}, \tau$ and $K$ settings. The same values have been assigned to all controllers. A star indicates a combination leading to an acceptable post-disturbance evolution, while a dot indicates failure to save the system. The criterion to accept a post-disturbance evolution was that all transmission voltages remain above $0.70 \mathrm{pu}$.

It can be seen that for $V^{\text {th }}=0.89$ or $0.90 \mathrm{pu}$, the controllers succeed saving the system for a large set of $(\tau, K)$ combinations. On the other hand, for $V^{\text {th }} \leq 0.88 \mathrm{pu}$, the voltages drop so fast that the controllers have no time to act, no matter the $(\tau, K)$ combination. Indeed, in most cases, the monitored transmission voltages drop from $V=V^{t h}$ (controller started) to $V=0.70 \mathrm{pu}$ (evolution rejected) in less time than the activation delay $\tau$. Thus, the range of acceptable $V^{t h}$ values appears to be very narrow.

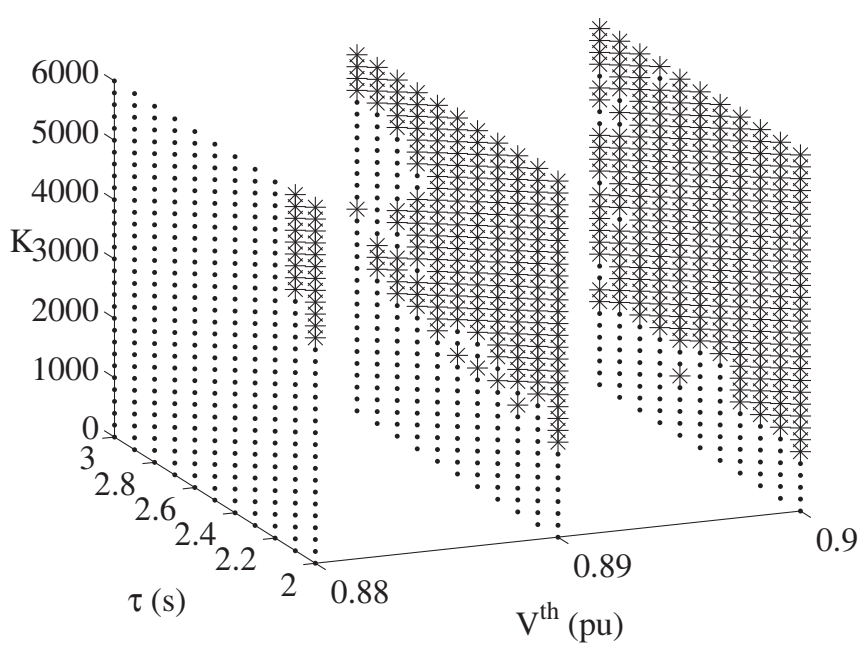

Fig. 10. Performance of distributed load shedding scheme for various settings (post-disturbance voltages refused below $0.70 \mathrm{pu}$ )

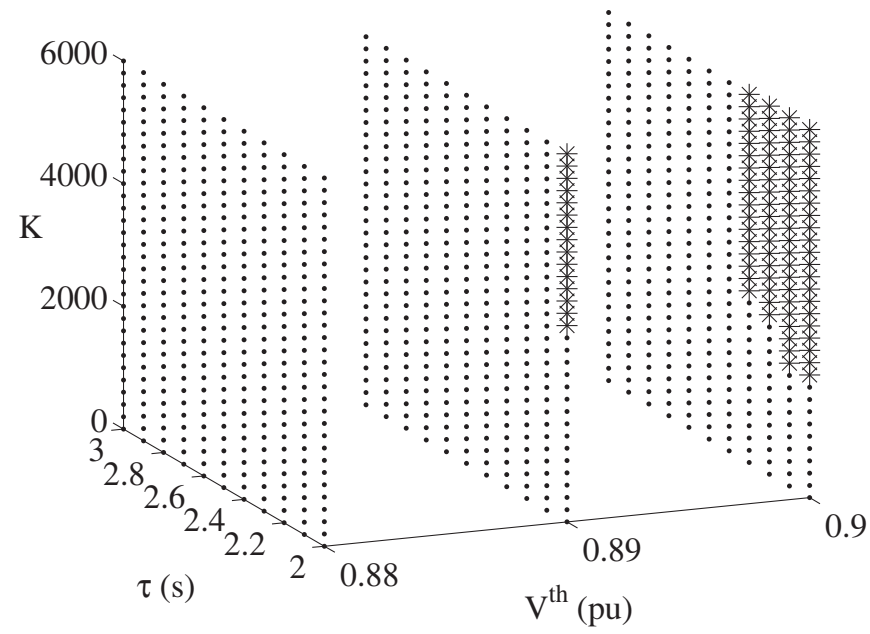

Fig. 11. Performance of distributed load shedding scheme for various settings (post-disturbance voltages refused below $0.80 \mathrm{pu}$ )

Furthermore, if post-disturbance evolutions are refused when voltages fall below $0.80 \mathrm{pu}$, as considered in [9], the diagram of successful $\left(V^{\text {th }}, \tau, K\right)$ combinations becomes the one in Fig. 11. Although for $V^{t h}=0.90$ pu a significant number of $(\tau, K)$ combinations still yield an acceptable system evolution, this protection scheme is not acceptable from the reliability viewpoint. In such a situation, it may be appropriate to resort to the wide-area protection presented in Section II-C, whose results are presented in the next section.

\section{Simulation Results: Wide-AREA SCHEME}

\section{A. Setting the controller parameters}

According to the wide-area protection scheme of Section II-C, the shedding delay is reduced to $\tau^{r e d}$ upon receipt of the overexcitation signal from one of the neighbouring generators. Generators g15, g14 and g12 are good candidates in view of their response to disturbances endangering voltage stability (see Fig. 6). 


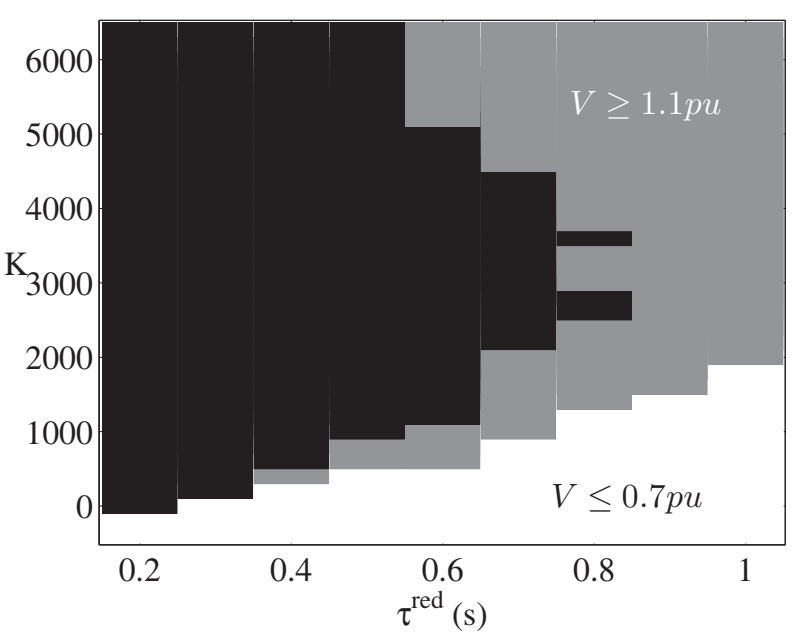

Fig. 12. Regions of successful, unsuccesful, and overvoltage operation of the wide-area protection

$\tau^{\text {red }}$ can be set to a low value, typical of undervoltage load shedding against short-term voltage instability: see $\tau_{S T}^{\min }$ in Fig. 2. Tests have been performed with $\tau^{\text {red }}$ in the interval $\left[\begin{array}{ll}0.2 & 1\end{array}\right] \mathrm{s}$.

Tests similar to those reported in Figs. 10 and 11 have shown that the wide-area protection is much less sensitive to the value of $V^{t h}$. Therefore the latter has been set to $0.85 \mathrm{pu}$, which leaves a more comfortable margin with respect to undesired operation. The results shown in the sequel have been obtained for that voltage threshold.

Finally, it has been found that the choices of $\tau^{r e d}$ and $V^{t h}$ are not tightly bound to each other as in the distributed scheme.

The criterion to accept a post-disturbance evolution is that all transmission voltages remain in the interval [0.70 1.10] pu. The upper limit was added to avoid overvoltages as a result of dropping off too much load in a very short time interval.

Figure 12 shows three regions of operation of the protection scheme in the $\left(\tau^{r e d}, K\right)$ space. Again, the same values have been assigned to all controllers. The disturbance of concern is the one already considered in Figs. 5, 6 and 8. The black region corresponds to proper operation yielding voltages within the prescribed limits. The white region corresponds to failure to stabilize the system. Finally, the grey region corresponds to $\left(\tau^{r e d}, K\right)$ combinations for which the system is stabilized but experiences overvoltages. Although not desirable from operation viewpoint, it is of course preferable to the white area.

The selectivity of the wide-area protection scheme, in terms of amount of load power shed, is illustrated in Fig. 13, showing the total load power shed for the various $\left(\tau^{r e d}, K\right)$ combinations inside the black region in Fig. 12. As expected, choosing a larger $\tau^{\text {red }}$ requires choosing a larger $K$, leading to more load shedding. As quoted in [8], the zones of equal shedding are not limited by smooth boundaries. This is due to the discrete nature of the controllers: a small change in $\tau^{\text {red }}$ or $K$ may lead to a smaller or larger load shedding by one controller, which in turn influences the neighbouring controllers.

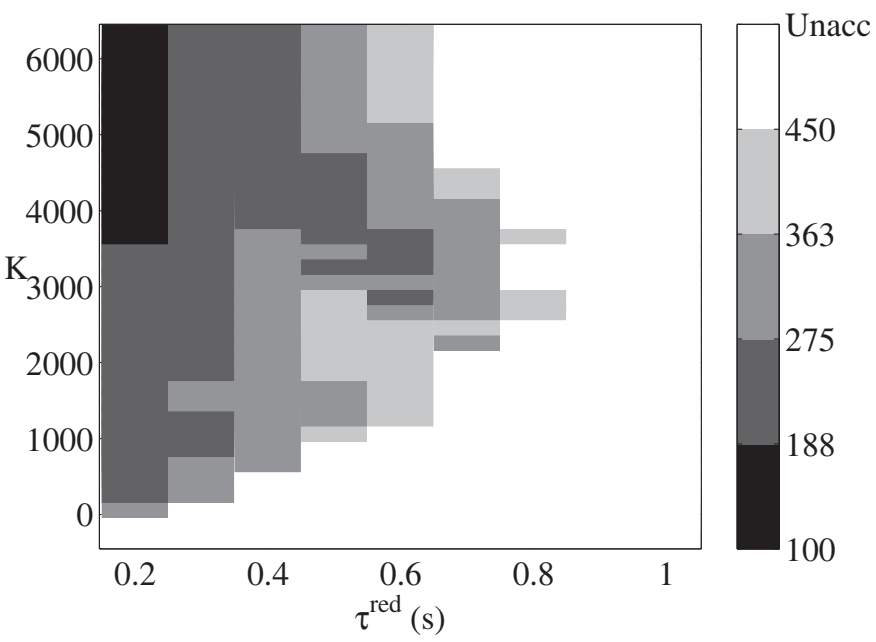

Fig. 13. Total power shed (in MW) by the wide-area protection for various $\left(\tau^{r e d}, K\right)$ combinations
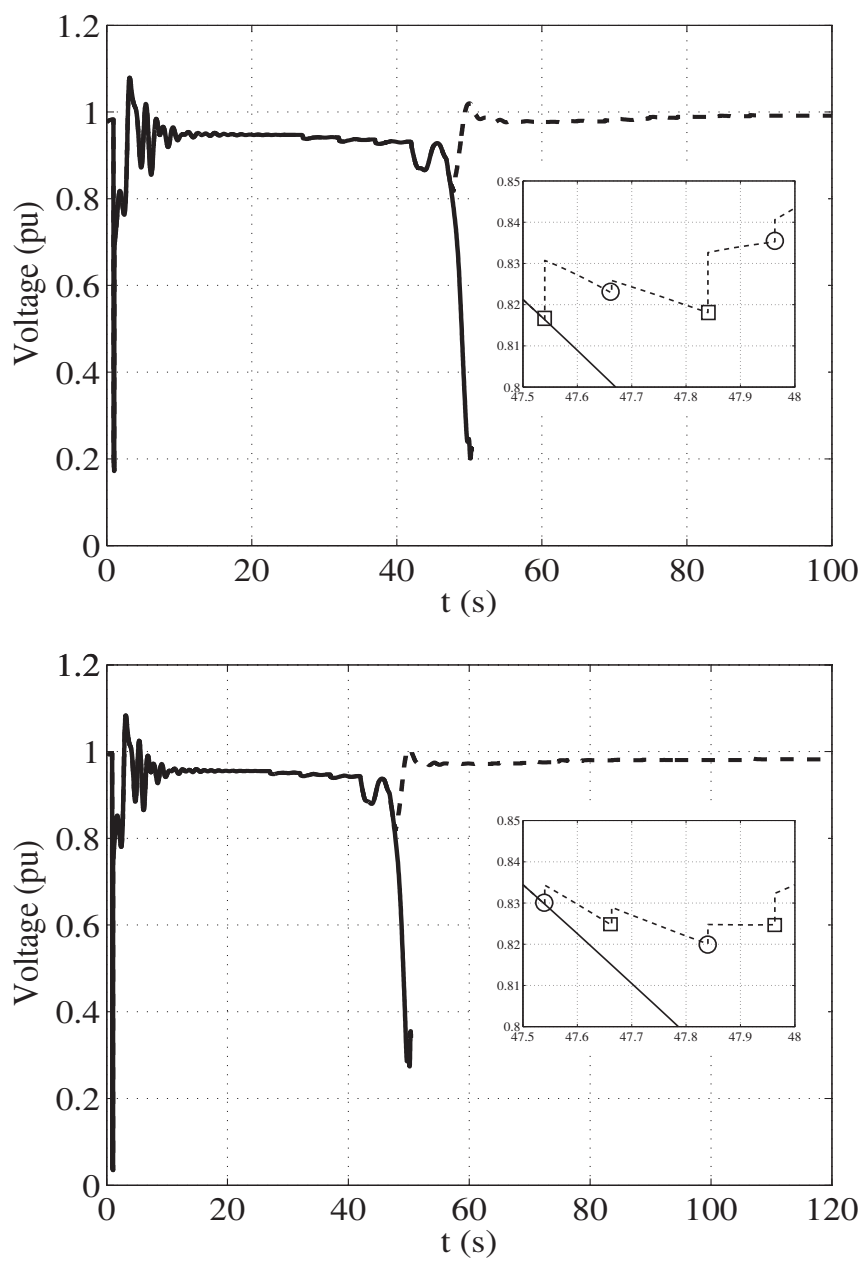

Fig. 14. Monitored voltage and shedding actions by controllers $C_{1041}$ (upper plot) and $C_{1044}$ (lower plot)

\section{B. Example of cooperation between controllers}

This section illustrates how the controllers cooperate into saving the system. All controllers have $\tau^{\text {red }}=0.3 \mathrm{~s}$ and $K=3000 \mathrm{MW} / \mathrm{pu}$. Figure 14 refers to controllers $C_{1041}$ and 
$C_{1044}$ (see Table I), which both react to the disturbance. The curves shown with solid lines are the unstable voltage evolutions observed at the controllers' transmission buses in the absence of load shedding, while the dashed lines correspond to the system stabilized by these controllers. The figure also offers a detailed view of the time interval in which shedding takes place. The squares indicate actions by the controller of concern while the circles indicate actions by the other controller.

As can be seen, both controllers act twice with a time delay of $0.3 \mathrm{~s}$ between sheddings. After the last shedding by $C_{1044}$, the cumulated actions of both controllers make the monitored voltages recover above $V^{t h}=0.85 \mathrm{pu}$ and the controllers return to idle state.

\section{Robustness with respect to component failure}

Figure 14 shows that the successive sheddings take place in a very short time interval (approximatively $0.4 \mathrm{~s}$ ). In such conditions, the robustness of the proposed scheme with respect to possible controller failures needs to be checked. An illustration is given in Table II showing the power shed by each controller in various failure scenarios.

TABLE II

LOAD SHEDDING AMOUNTS (IN MW) IN VARIOUS SCENARIOS

\begin{tabular}{|c||c|c|c|c|c|}
\hline Controller & Case 1 & Case 2 & Case 3 & Case 4 & Case 5 \\
\hline$C_{1041}$ & 130 & - & - & - & - \\
\hline$C_{1042}$ & 0 & 0 & 0 & 0 & - \\
\hline$C_{1043}$ & 0 & 67 & 115 & 115 & - \\
\hline$C_{1044}$ & 94 & 150 & - & - & 370 \\
\hline$C_{1045}$ & 0 & 88 & 250 & - & - \\
\hline \hline Total & 224 & 305 & 365 & unstable & 370 \\
\hline
\end{tabular}

Case 1 corresponds to the simulation shown in Fig. 14.

Case 2 simulates a full failure of $C_{1041}$, the controller with the largest action in Case 1. This is compensated by the stronger action of $C_{1044}$ and the activation of $C_{1043}$ and $C_{1045}$.

Similarly, Case 3 corresponds to failure of both $C_{1041}$ and $C_{1044}$, leading to an even larger shedding by $C_{1043}$ and $C_{1045}$.

In Case 4 the failure of $C_{1045}$ is added to the situation of Case 3. The system cannot be stabilized by the remaining two controllers. Indeed, on one hand, $C_{1043}$ sheds the whole interruptible part of its load but this amounts to $115 \mathrm{MW}$ (see Table I), which is not enough. On the other hand, $C_{1042}$ is never activated because, being located near generator g6 (see Fig. 4), the voltage it monitors remains above $V^{t h}$.

To support the above explanation, Case 5 shows a situation where $C_{1044}$ alone succeeds stabilizing the system, thanks to a larger interruptible load power.

As expected, the total power shed is increasing as the remaining controllers compensate for the missing ones, which would have responded first to the voltage drops. Nevertheless, the redundancy among controllers makes the proposed protection scheme very reliable.

\section{Conclusion}

In this paper, the implementation of undervoltage load shedding to counteract long-term voltage instability in the presence of induction motor loads has been investigated and improved.

The possible limitations of the purely distributed protection scheme previously proposed by the authors have been analyzed and illustrated from time simulations of a small but representative and stringent test system. Simply stated, to preserve security of the protection, this undervoltage-based scheme lacks some speed of action, which endangers its dependability.

To reconcile these two important features, an extension bearing the spirit of wide-area protection has been considered. The idea is to send information about generator overexcitation to the distributed controllers, to allow them shedding faster in case of voltage drop. Without this authorization, the controllers keep on acting with a longer delay in order for them not to react to a normally cleared fault. Successful results have been obtained on the same test system.

The improved behaviour is obtained at the expense of a moderate increase in complexity, and hence little exposure to failure. Moreover, the important features of the simple distributed scheme such as closed-loop operation and redundancy between controllers are preserved.

\section{REFERENCES}

[1] C. W. Taylor,"Concepts of undervoltage load shedding for voltage stability", IEEE Trans. on Power Delivery, Vol. 7, 1992, pp. 480-488.

[2] S. Arnborg, G. Andersson, D.J. Hill, I.A. Hiskens, "On undervoltage load shedding in power system," Int. Journal of Electrical Power and Energy Systems, vol. 19, 1997, pp. 141-149.

[3] D. H. Karlsson (convener), System Protection Schemes in Power Networks, Final report of CIGRE Task Force 38.02.19, June 2001.

[4] V.C. Nikolaidis, C.D. Vournas,"Design Strategies for Load-Shedding Schemes against Voltage Collapse in the Hellenic System," IEEE Trans. Power Syst., Vol. 23, No. 2, May 2008.

[5] B. Ingelsson, P. Lindström, D. Karlsson, G. Runvik, J. Sjödin, "Special Protection Scheme against Voltage Collapse in the South Part of the Swedish Grid," Proc. CIGRE Conference, 1996, Paper No 38-103.

[6] D. Lefebvre, C. Moors, T. Van Cutsem, "Design of an undervoltage load shedding scheme for the Hydro-Québec system", Proc. IEEE PES General Meeting, Toronto (Canada), July 2003.

[7] S. Imai, "Undervoltage load shedding improving security as reasonable measure for extreme contingencies", Proc. IEEE PES General Meeting, San Francisco (USA), 2005.

[8] B. Otomega, T. Van Cutsem, "Undervoltage load shedding using distributed controllers," IEEE Trans. Power Syst., vol. 22, no. 4, pp. 1898-1907, Nov. 2007.

[9] F. Capitanescu, B. Otomega, H. Lefebvre, V. Sermanson, T. Van Cutsem "Prospects of an improved system protection scheme against voltage instability in the RTE system", Proc. 16th Power System Computation Conference (PSCC), Glasgow (UK), June 2008, ISBN/EAN:978-0947649-28-9.

[10] C. W. Taylor, Power System Voltage Stability, EPRI Power System Engineering Series, McGraw Hill, 1994.

[11] T. Van Cutsem, C. Vournas, Voltage Stability of Electric Power Systems, Boston, Kluwer Academic Publishers (now Springer), 1998.

[12] C. Vournas, T. Van Cutsem, "Local identification of voltage emergency situations," IEEE Trans. Power Syst., vol. 23, no.1 , pp. 1239-1248, Feb. 2008.

[13] M. Glavic, T. Van Cutsem, "Detecting with PMUs the onset of voltage instability caused by a large disturbance," in Proc. 2008 IEEE Power Eng. Soc. General Meeting, Pittsburgh, PA, Jul. 2008. 